\title{
Exploring the non-equilibrium fluctuation relation for quantum mechanical tunneling of electrons across a modulating barrier
}

\author{
Dibya J. Sivananda, Nirmal Roy, P. C. Mahato, and S. S. Banerjee $\odot^{*}$ \\ Department of Physics, Indian Institute of Technology Kanpur, Kanpur 208016, India
}

(Received 31 January 2020; accepted 13 October 2020; published 16 November 2020)

\begin{abstract}
We study the validity of the fluctuation relation for a non-equilibrium quantum mechanical system, viz., electrons quantum mechanically tunneling across a periodically modulating barrier. Experimentally this system is realized by measuring the fluctuation in the tunneling current between an STM tip and a vibrating gold film deposited on a piezo crystal. The long time series of tunneling signal shows large positive and negative fluctuations. The analysis shows that over finite observation time intervals, the probability distribution of the average rate of work done has a positive mean. About this mean, the distribution is broad and it is spread over not only positive but also negative values. These positive and negative values correspond to work done either on the electron by the external drive or work done by the electron against the drive, as it tunnels across the modulating barrier, respectively. For different driving frequencies, we show that the probability distribution satisfies the non-equilibrium fluctuation relation (NEFR). Thus, we prove that the NEFR is valid for a driven quantum mechanical system. For this tunneling process, we determine the large deviation function (LDF), which is related to NEFR. We see changes in the shape of the LDF as a function of the drive frequency, although NEFR is valid at all these frequencies. Measuring dissipation associated with microscopic irreversible trajectories in non-equilibrium quantum mechanical systems is a challenging task. Here we use NEFR also to obtain a measure of the dissipation associated with the electron tunneling across the modulated barrier.
\end{abstract}

DOI: 10.1103/PhysRevResearch.2.043237

\section{INTRODUCTION}

For systems in equilibrium, there is no net heat exchanged between the system and the environment. However, for driven non-equilibrium (NEQ) steady-state systems, the applied drive helps to overcome the energy lost from the system as the dissipated energy flows to the surrounding environment. However, unlike macroscopic systems, smallsized driven systems show unusual features, due to their high susceptibility to fluctuation effects which can irreversibly modify the trajectories along which the system evolves. For example, consider a small particle being driven through a viscous medium. Within a finite time window, heat dissipated from the driven system is transferred to the surrounding environment (medium). However, occasionally, this particle can also capture some heat from the environment [1,2], which irreversibly modifies the trajectory of the small particle. Consequently, for such systems, repeated measurements on an NEQ system prepared with identical protocol over a finite observation time window shows a probability distribution of the heat exchanged between the system and the environment. The heat exchanged can be both positive and negative, de-

\footnotetext{
*satyajit@iitk.ac.in

Published by the American Physical Society under the terms of the Creative Commons Attribution 4.0 International license. Further distribution of this work must maintain attribution to the author(s) and the published article's title, journal citation, and DOI.
}

scribing the two-way flow leading to irreversible trajectories. For describing the behavior of such NEQ systems, one defines the average entropy production or consumption rate $\left(s_{\tau}\right)$, in a time interval $(\tau)$ as $s_{\tau}=\frac{1}{\tau} \int_{t}^{t+\tau} s\left(t^{\prime}\right) d t^{\prime}$. The probability distribution of $s_{\tau}$, viz., $P\left(s_{\tau}\right)$, is spread over $s_{\tau}$ values which are both positive (entropy increasing events) as well as negative (entropy consuming events). The $P\left(s_{\tau}\right)$, satisfies some useful mathematical relations, viz., the non-equilibrium fluctuation relations (NEFRs). The NEFR, seen in simulations by Evans and Searles [3] and then proved by Gallavotti and Cohen [4], is for non-equilibrium steady-state systems with timeindependent driving forces. Subsequently, Shragel and Chou [5] derived a general form of NEFR for situations involving time-dependent driving forces to account for forward $(f)$ and reverse $(r)$ paths of the system. This general form of NEFRs is

$$
\operatorname{Lt}_{\tau \rightarrow \infty} \frac{P^{f}\left(+s_{\tau}\right)}{P^{r}\left(-s_{\tau}\right)}=e^{\tau s_{\tau}} .
$$

Equation (1) states that within a duration $\tau$, the probability of the positive time-averaged entropy production rate for the forward path $\left[P^{f}\left(+s_{\tau}\right)\right]$ is exponentially larger than the probability of entropy consumption rate for the reverse path $\left[P^{r}\left(-s_{\tau}\right)\right]$. Typically, for macroscopic systems $P^{f}\left(+s_{\tau}\right)$ dominates. However, in different NEQ situations, like for driven small-sized systems, the $P^{r}\left(-s_{\tau}\right)$ is also significant. For a system with symmetric forward and reverse drive, the $f$ and $r$ superscripts in Eq. (1) are dropped.

The Gallavotti-Cohen non-equilibrium fluctuation relation (NEFR) has been verified for a diverse variety of driven 
systems, like sheared micellar gels in a jammed state [6], a mechanically driven fluidized medium of inelastic beads [7], dragging of a Brownian particle using an optical trap [8,9], electrical circuits [10], RNA stretching, Rayleigh-Bernard convection [11,12], pressure fluctuations on the surface kept in a turbulent flow [13], vertically shaken granular beads [7], Lagrangian turbulence on a free surface [14], liquid crystal electro-convection [15], vortices in superconductors [16], driven levitating nanoparticles [17], etc. While these verifications of NEFR have been demonstrated predominantly for classical driven systems, in our work, we explore the validity of NEFR for a periodically driven NEQ quantum mechanical system. Specifically, we are interested in studying NEFR in the context of an electron tunneling across a modulating potential barrier. While quantum mechanical tunneling across a static barrier is a popular undergraduate textbook topic [18], the study of tunneling across a periodically modulated barrier is a complex problem. In such systems, the tunneling across the modulated barrier itself affects the evolution of the tunneling wave [19-22]. Such systems involve the exchange of energy between the system and the barrier and such open quantum systems cannot be studied using Schrodinger's equations [23-26]. In fact, in recent times, new areas have emerged in this field, like dissipative phase transition in open systems [27-32]. While dissipation is important, experimentally measuring it for a NEQ quantum mechanical system is a challenging task.

Recently we have shown [33] that tunneling between the scanning tunneling microscope (STM) tip and a conducting surface (Au film) on a piezo electric crystal vibrating at a frequency $\omega$, produces a modulation in the tunneling current at a frequency $\omega$. The modulation in the tunneling current is because of the modulation in the tunneling barrier at frequency $\omega$. In the present work, we report observing large positive and negative fluctuations in the long time series of the tunneling signal. Over finite observation time intervals, the probability distribution of the average rate of work done has a positive mean. About this mean, the distribution is broad and spreads over significantly large values which are positive as well as a negative value. The positive and negative fluctuations are related to the work done either on the electron by the external drive or by the tunneling electron against the drive, respectively. We show that the probability distribution of this work satisfies the NEFR for a driven quantum mechanical tunneling system. While most earlier studies have been for driven classical systems, this demonstration of validity of NEFR in the quantum regime uses a quantum mechanical tunneling system. We also determine the large deviation function (LDF), which is associated with the probability of observing large fluctuation events in a non-equilibrium system and is closely related to NEFRs. We also use the NEFR to obtain a measure of the dissipation for the electron tunneling across a barrier, which is modulated at different frequencies.

\section{EXPERIMENTAL DETAILS}

We use a Quazar Technologies room-temperature STM (NanoRev. 4.0). In Fig. 1(a), we show the atomic arrangement in a highly ordered pyrolytic graphite (HOPG) sample imaged using this STM. We placed below the STM tip a conducting gold film deposited on top of a vibrating piezoelectric crystal which has a diameter of $\sim 1.4 \mathrm{~cm}$ and thickness of $\sim 0.33 \mathrm{~mm}$. The piezo is stuck to a glass substrate $(1 \mathrm{~cm} \times 2 \mathrm{~cm})$ which is then stuck to the gold-coated metallic stub of the STM with a double-sided adhesive tape [see Fig. 1(a)]. The STM circuit is completed by shorting the top conducting surface of the piezoelectric crystal with the gold-coated STM metallic stub. For our STM tip, we use an electrochemically etched Pt-Ir alloy wire and maintain a constant dc bias $V_{b}=-1.5 \mathrm{~V}$ between the tip and the gold film on top of the vibrating piezo surface. The STM in our experiment is operated in constant current mode. The tunneling current modulations can be measured from the voltage drop across a 1-G $\Omega$ resistor $\left(V_{T}=I_{\mathrm{TC}} \times 10^{9} \Omega\right)$ using a DSO (digital storage oscilloscope). In our measurement, the time series of the feedback signal $\left[V_{\mathrm{FB}}(t)\right]$ from the STM is measured using a DSO (Yokagawa DL 9000 series) with a sampling rate of 5 Giga-samples per second. In order to avoid any ac electrical coupling between the bottom surface of the piezo (an oscillating voltage is applied to the bottom surface to vibrate the piezo) with the piezo's top surface, the top surface of the piezo crystal [Fig. 1(a)] is grounded through a $10-\mu \mathrm{F}$ polar capacitor.

Using this setup under identical ambient conditions, we measure the time series of the tunneling currents between the STM tip and the vibrating gold film on the surface of a piezo crystal which is vibrating with frequency $f$. The piezo crystal is vibrated with a sinusoidal ac voltage with a peak to peak amplitude of $30 \mathrm{~V}$ and a frequency $f$. The tunneling current has an exponential dependence on the tip to surface distance. Hence the periodically changing tunneling spacing between the STM tip and the vibrating Au surface [see schematic in Fig. 1(b)] produces modulation in the tunneling current. The modulation in the tunneling current (equivalently in the STM feedback $V_{\mathrm{FB}}$ signal) is at the same frequency with which the piezo is vibrated, viz., at a few 100's $\mathrm{kHz}$. These periodic variations of the tunneling current at the frequency $f$ with which the piezo crystal is vibrated has been shown before in Ref. [33]. Thus the $V_{\mathrm{FB}}$ signal enters a sensitive detectable regime which is well above the noise floor of the STM electronics. Here we begin to sensitively detect the intermittent large fluctuations in the tunneling signal.

In the constant current mode of STM operation, we measure the long time series of the STM feedback signal $\left(V_{\mathrm{FB}}\right)$. The $V_{\mathrm{FB}}$ is directly proportional to the tunneling signal $\left(V_{T}\right)$, (see Appendix A), which shows the linear relationship between the measured $V_{\mathrm{FB}}$ and $V_{T}$ ). The time series of the feedback signal $\left[V_{\mathrm{FB}}(t)\right]$ was captured for different $f$ of the vibrating piezo crystal, ranging from 100 to $1000 \mathrm{kHz}$. The frequency response of our STM circuit shows significant attenuation only beyond $10 \mathrm{MHz}$ [see the frequency response of our STM in Fig. 5(c) of Ref. [33]]. Initially, before modulating the piezo surface, the mean value of $V_{\mathrm{FB}}$ is determined. This means the $V_{\mathrm{FB}}$ value is proportional to the average value of the tunneling current established between the tip and the non-vibrating conducting gold film surface. In all subsequent figures, this mean $V_{\mathrm{FB}}$ value is subtracted from $V_{\mathrm{FB}}(t)$. Figures 1(c), 2(a), 2(d), and 2(g) show the long time series of $V_{\mathrm{FB}}(t)$ data captured (for $500 \mathrm{~s}$ ) for different $f$. In Fig. 1(c) the green data points show the fluctuations in $V_{\mathrm{FB}}(t)$ when the piezo crystal is vibrated at $500 \mathrm{kHz}$. The red data points 


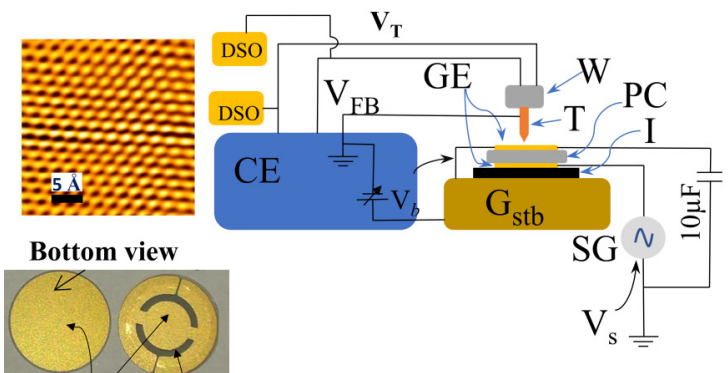

GE Top view

(a)

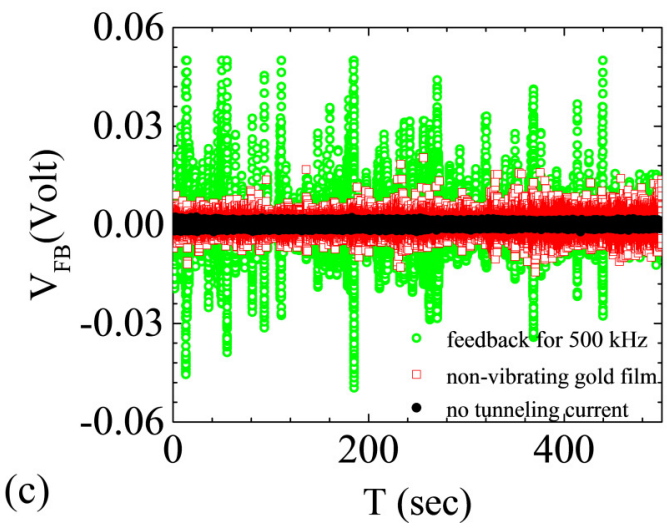

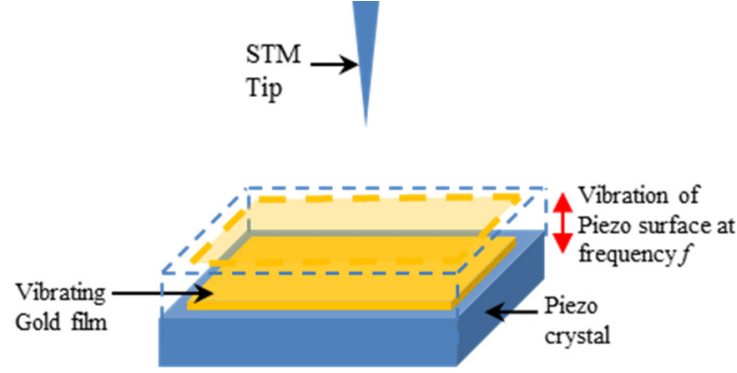

(b)

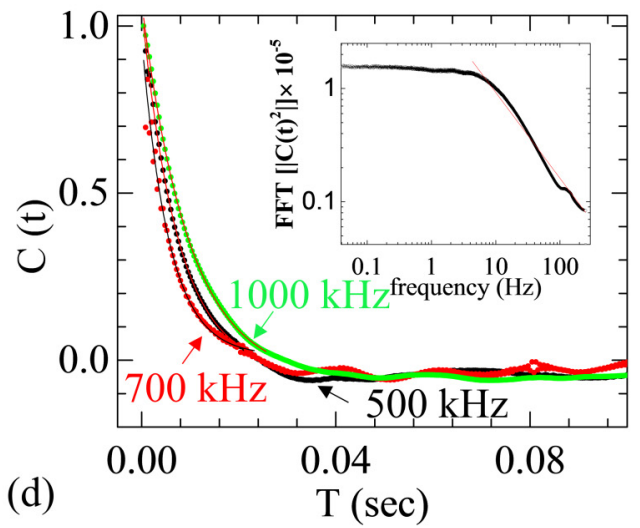

FIG. 1. (a) Atomic resolution image of a HOPG surface captured with our STM, schematic of the STM circuit, and the front and backside of the piezoelectric crystal used. The abbreviations used in the schematic are GE: gold electrodes; CE: STM control electronics; DSO: digital storage oscilloscope; W: STM piezo walker; T: STM tip; PC: piezo crystal below tip; I: insulating layer; SG: function signal generator; $\mathrm{G}_{\text {stb }}$ : gold coated stub; $\mathrm{V}_{\mathrm{FB}}$ : feedback signal of the $\mathrm{STM} ; \mathrm{V}_{\mathrm{T}}$ : tunneling voltage signal; $\mathrm{V}_{\mathrm{b}}$ : tip to sample bias voltage; $\mathrm{V}_{\mathrm{s}}$ : voltage signal from signal generator. (b) Schematic of the piezo vibrating beneath the STM tip. (c) The electronic noise of the STM (with the tunneling current on and off) is compared with the fluctuations in the $V_{\mathrm{FB}}$ when the piezo crystal is vibrating at $500 \mathrm{kHz}$. It shows that electronic noise is one order of magnitude smaller than the feedback signal when the piezo crystal is vibrating. (d) The autocorrelation function for the feedback voltage at different frequencies of the vibrating piezo. Inset shows the $\frac{1}{\mathrm{f}}$ noise of the 500-kHz signal [shown in Fig. 1(c) green points] in log-log scale.
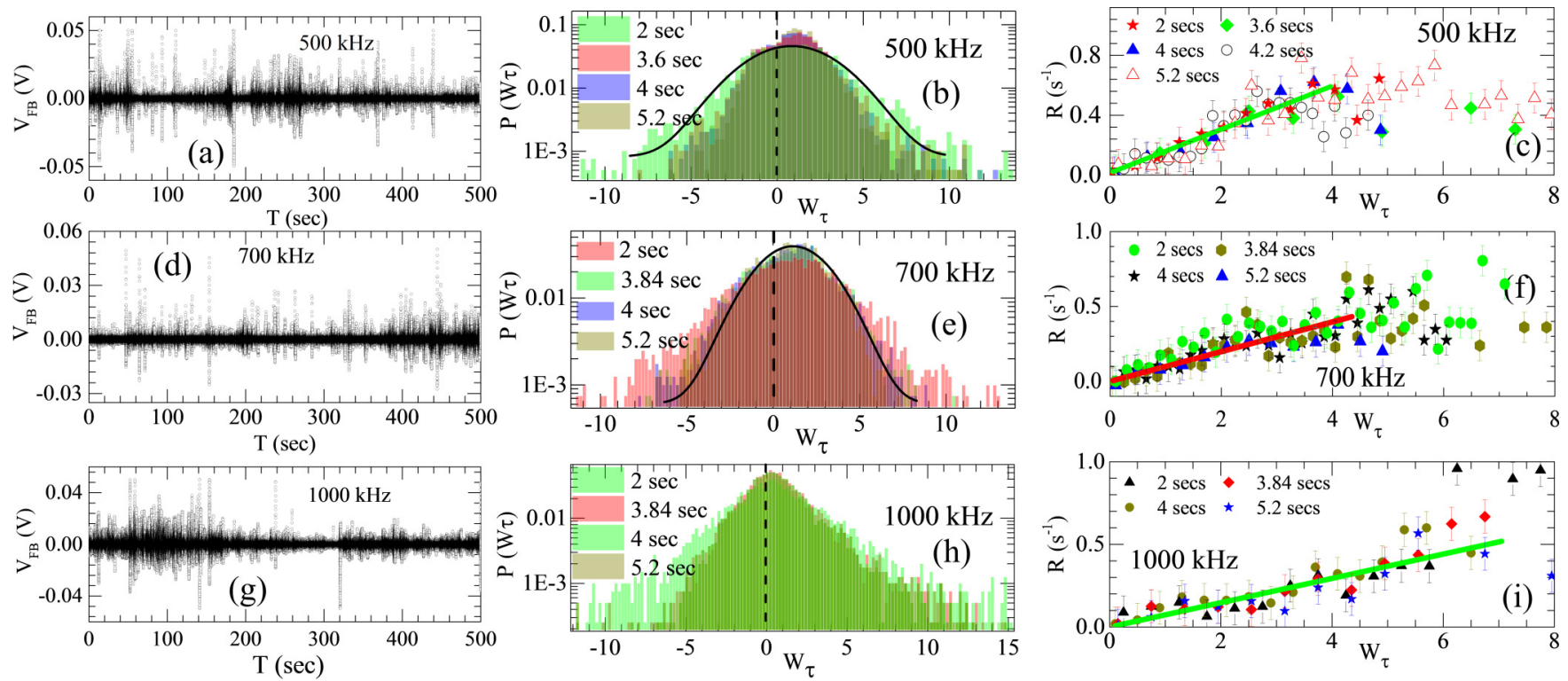

FIG. 2. (a) Time series of the feedback voltages for a frequency of $500 \mathrm{kHz}$ of the vibrating piezo. (b) The probability distribution functions (PDFs) of the time series in (a). (c) The $R$ vs $W_{\tau}$ for $500-\mathrm{kHz}$ frequency. (d) Time series of the feedback voltages for a frequency of $700 \mathrm{kHz}$ of the vibrating piezo. (e) The probability density functions of the time series in (d). (f) The $R$ vs $W_{\tau}$ for $700-\mathrm{kHz}$ frequency. (g) Time series of the feedback voltages for a frequency of $1000 \mathrm{kHz}$ of the vibrating piezo. (h) The probability density functions of the time series in (g). (i) The $R$ vs $W_{\tau}$ for $1000-\mathrm{kHz}$ frequency. 
show the fluctuations in $V_{\mathrm{FB}}(t)$ when the tip is positioned over a non-vibrating substrate. The black data points in Fig. 1(c) show the bare STM electronics noise floor voltage fluctuations when no tunneling current exists between the STM tip and the surface (viz., when the tip is retracted to a position far away from the gold surface). A comparison of the above signals shows that although the fluctuations in the $V_{\mathrm{FB}}(t)$ from a non-vibrating substrate (red data points) are above the STM electronics noise floor level (black points), the fluctuations in the red data points are an order of magnitude lower than that of the green data points. Also, for the non-vibrating case (red data points) the statistics of the fluctuations above the noise floor are far lower than that for the vibrating surface case (green data points).

Figure 1(d), main panel, shows the autocorrelation function [C( $t)]$ of $V_{\mathrm{FB}}(t)$ data [shown in Figs. 2(a), 2(d), and 2(g)] captured for $f=500,700$, and $1000 \mathrm{kHz}$. The $C(t)$ calculated using, $C(t)=\frac{\left\langle V_{\mathrm{FB}}\left(t+t^{\prime}\right) V_{\mathrm{FB}}(t)\right\rangle}{V_{0}^{2}}$ where $V_{0}$ is the mean of $V_{\mathrm{FB}}(t)$ and $\langle\ldots\rangle=\frac{1}{T} \int_{0}^{T} \ldots d t^{\prime}$ shows that the fluctuations in $V_{\mathrm{FB}}(t)$ signals are uncorrelated beyond tens of milliseconds. We would like to emphasize that the large fluctuations observed in the time series of the feedback signal [Fig. 1(c)] is not an artefact of a measurement technique. To show this consider the following: Had the observed fluctuations been an artefact of the measurement, then we should observe a strong correlation between the fluctuations with $f$. However quite contrary to this expectation, Fig. 1(d) shows, for $f$ varying from 500 to $1000 \mathrm{kHz}, C(t) \rightarrow 0$ by few tens of milliseconds, uniformly. Hence the fluctuations are intrinsic. Equation (1) is valid for this choice of time intervals $\tau$, over which the fluctuations are uncorrelated. For our subsequent NEFR analysis, the $C(t)$ analysis limits our choice of $\tau$ to be larger than a few tens of milliseconds. The inset of Fig. 1(d) shows the behavior of the Fourier transform of $|C(t)|^{2}$ for the $500-\mathrm{kHz}$ data [power spectrum $S(f)$ ] as a function of frequency on a log-log scale. The inset of Fig. 1(d) shows that the power spectrum of the noise has a non-shot-noise type behavior, viz. $S(f) \propto \frac{1}{f^{\alpha}}$ where $\alpha=0.7 \pm 0.1$ [recall that the Flicker noise is characterized by $\left.S(f) \propto \frac{1}{f}\right]$. The absence of the shot-noise feature suggests that the large fluctuations in tunneling signal are not related to the discreet nature of the tunneling process, viz., discrete electronic charges tunneling across the barrier. Clearly, the long time series picks up large fluctuation events for electrons tunneling across the modulating tunneling gap.

We would like to mention here that as we are using a symmetric, sinusoidal drive, the forward and reverse drives are symmetric, and hence we drop the superscripts $f$ and $r$ in Eq. (1). In Fig. 2(a) we show the $V_{\mathrm{FB}}(t)$ signal for $f=$ $500 \mathrm{kHz}$. In Fig. 2(b) we analyze the time series in Fig. 2(a) in terms of $P\left(W_{\tau}\right)$. Here the $P\left(W_{\tau}\right)$ is the probability of observing an event of magnitude $W_{\tau}$ within an observation time interval of $\tau$. For our analysis, $V_{\mathrm{FB}}(t)$ is broken up into a series of time bins each of width $\tau$ where we calculate $W_{\tau}$ as per Eq. (2),

$$
W_{\tau}=\frac{s_{\tau}}{\langle s(t)\rangle}=\frac{\frac{1}{\tau} \int_{t}{ }^{t+\tau} I_{T}\left(t^{\prime}\right) V_{b} d t^{\prime}}{V_{b}\left\langle I_{T}\right\rangle} .
$$

Here $I_{T}$ is the tunneling current, and $V_{b}$ is the constant dc STM bias voltage applied between the STM tip and base. Note that $I_{T} \propto V_{T}$ where $V_{T}$ is the tunneling voltage and $V_{\mathrm{FB}} \propto V_{T}$ (see
Appendix A). Therefore,

$$
W_{\tau}=\frac{s_{\tau}}{\langle s(t)\rangle}=\frac{\frac{1}{\tau} \int_{t}{ }^{t+\tau} V_{\mathrm{FB}}\left(t^{\prime}\right) V_{b} d t^{\prime}}{V_{b}\left\langle V_{\mathrm{FB}}\right\rangle},
$$

where $\left\langle V_{\mathrm{FB}}\right\rangle$ is the average value of the $V_{\mathrm{FB}}(t)$ signal within an observation time interval $\tau . W_{\tau}$ is the average work done within the time interval $\tau$, for electrons tunneling between the STM tip and the vibrating Au film on the piezo crystal's surface.

Often for experimental studies, the non-equilibrium fluctuation relation (NEFR) of Eq. (1) is restated in terms of $W_{\tau}$ $[6,7]$ as

$$
R=\frac{1}{\tau} \ln \left(\frac{P\left(+W_{\tau}\right)}{P\left(-W_{\tau}\right)}\right)=s_{\tau}=W_{\tau}\langle s(t)\rangle .
$$

Here, $s(t)=I V / k_{B} T_{\text {eff }}[6,7,15]$, where $I(t) V$ is the instantaneous power flux into the system, and $T_{\text {eff }}$ is an effective temperature scale for the NEQ system, and it is not related to the equilibrium temperature of the system. Note that for proving the validity of the NEFR for our quantum mechanical tunneling situation, as per Eq. (4), we should demonstrate a linear relationship between $R$ versus $W_{\tau}$ with a unique slope $[=\langle s(t)\rangle]$ whose value should not depend on the choice of $\tau$. The $T_{\text {eff }}$ is calculated from $\langle s(t)\rangle$, where

$$
\langle s(t)\rangle=\langle I V\rangle / k_{B} T_{\text {eff }} .
$$

Here $I \sim 10^{-12} \mathrm{~A}, V=1.5 \mathrm{~V}$, and the slope of the $R$ versus $W_{\tau}$ curve is calculated from Fig. 2 .

From the long time series of the $V_{\mathrm{FB}}(t)$ signal in Fig. 2(a) we determine $W_{\tau}$ (for different choices of $\tau$ ) using Eq. (3). Note that the $\tau$ values are larger than the time interval of a few tens of milliseconds beyond which $C(t) \rightarrow 0$ [recall Fig. 1(d)], as discussed earlier. We then determine the probability distribution of $W_{\tau}$ values, viz., $P\left(W_{\tau}\right)$. The $P\left(W_{\tau}\right)$ versus $W_{\tau}$ curve in Fig. 2(b) for each $\tau$ has a Gaussian nature (the solid line is the Gaussian envelope) for small $W_{\tau}(\leqslant 4)$, but the distribution deviates from the Gaussian curve for larger $W_{\tau}$. The distribution has a peak near a $\left\langle W_{\tau}\right\rangle$ value of $\sim 1.1$. An important feature of the $P\left(W_{\tau}\right)$ distribution is that although it has a positive mean, it shows that along with positive work events there also exists a significant probability of negative work events (i.e., $-W_{\tau}$ events). While the positive $W_{\tau}$ events represent the usual entropy increasing events, the significant probability of the occurrence of the unusual negative $-W_{\tau}$ events represent tunneling electron trajectories which do work against the bias drive. These negative events are the characteristic signatures of the non-equilibrium irreversible trajectories discussed earlier. All of the above features, which are seen in Fig. 2(b) for $f=500 \mathrm{kHz}$ excitation, are also seen at $f=700$ and $1000 \mathrm{kHz}$ as well [see Figs. 2(e) and 2(h)].

Figure 2(c) shows a plot of $R$ versus $W_{\tau}$ for different choices of $\tau$, for the $500-\mathrm{kHz}$ data. We see a linear relation between $R$ versus $W_{\tau}$ for $0<W_{\tau} \leqslant 4$. We also see that for different choices of $\tau$, all the ( $R$ versus $W_{\tau}$ ) data scale onto one single curve which doesn't depend on $\tau$, and this is consistent with the NEFR relation in Eq. (4). To justify the scaling of the $R$ versus $W_{\tau}$ data, in Appendix B we have shown the unscaled behavior if one does not plot $R$ versus $W_{\tau}$. In Fig. 2(c) the best-fit curve through the data points (within the error bars), 

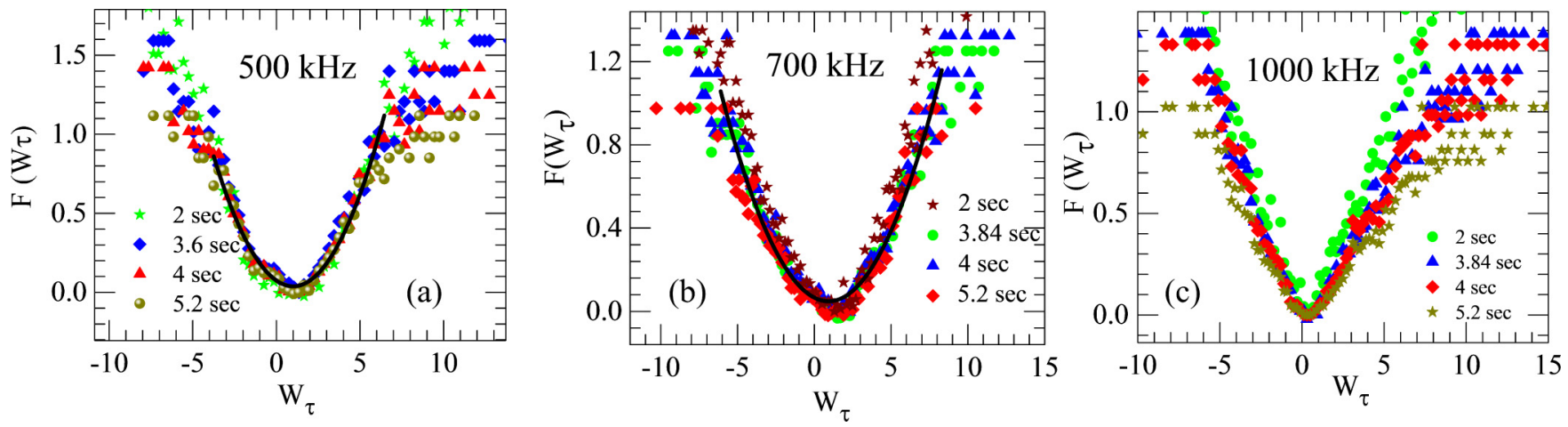

FIG. 3. $F\left(W_{\tau}\right)$ curves for different $\tau$ for (a) $500 \mathrm{kHz}$, (b) $700 \mathrm{kHz}$, and (c) $1000 \mathrm{kHz}$ of the vibrating piezo. Solid (black) line represents the data fitted with a form $a_{0}\left(W_{\tau}-\left\langle W_{\tau}\right\rangle\right)^{2}$, where the constant $\mathrm{a}_{0}$ is $0.033(500 \mathrm{KHz}), 0.020(700 \mathrm{kHz})$, and $\left\langle W_{\tau}\right\rangle=1.1 \mathrm{for} 500 \mathrm{kHz}$ and $700 \mathrm{kHz}$. At $1000 \mathrm{kHz}$ the $F\left(W_{\tau}\right)$ cannot be fitted to a quadratic form.

is a straight line with a unique slope [this is shown as the solid green line in Fig. 2(c)]. A similar validation of NEFR as per Eq. (4), for $f=700$ and $1000 \mathrm{kHz}$ is seen in Figs. 2(f) and 2(i). From Figs. 2(c), 2(f), and 2(i) we see that for $W_{\tau}$ values greater than 4 to 5 , the linear relation between $R$ and $W_{\tau}$ no longer holds, and we also observe the breakdown of the scaling feature of the data. Note that past experiments on classical driven systems have also validated the NEFR [Eq. (4)] within a limited range of $W_{\tau}[6,7,15-17]$. The above is an experimental validation of NEFR for a non-equilibrium process involving quantum mechanical tunneling across a periodically modulated barrier. The validity of NEFR indirectly shows that the tunneling trajectories across the modulating barrier are irreversible.

For these fluctuations in work, we try and determine the large deviation function (LDF), which is a measure of the probability of observing large fluctuation events, where the value of an observable is much greater than the mean value of the observable $[34,35]$. The NEFR is closely related to the LDF, viz., it is related to the symmetry property of the LDF $[34,35]$. To analyze the shapes of the $P\left(W_{\tau}\right)$ curves in Figs. 2(b), 2(e), and 2(h) we plot the LDF function $F\left(W_{\tau}\right)$ $[6,16,34,35]$ which is determined using

$$
F\left(W_{\tau}\right) \propto-\ln \left[P\left(W_{\tau}\right)\right] .
$$

If $P\left(W_{\tau}\right)$ has a Gaussian form then, as per Eq. (6), $F\left(W_{\tau}\right) \propto\left(W_{\tau}-\left\langle W_{\tau}\right\rangle\right)^{2}$ viz., $F\left(W_{\tau}\right)$ has a quadratic dependence on $W_{\tau}$. Using the $P\left(W_{\tau}\right)$ data of Fig. 2(b) and Eq. (6) we determine $F\left(W_{\tau}\right)$ by taking the natural logarithm of $P\left(W_{\tau}\right)$ [see Fig. 3(a)]. The solid line in Fig. 3(a) shows that $F\left(W_{\tau}\right)$ behaves as $\left(W_{\tau}-\left\langle W_{\tau}\right\rangle\right)^{2}$ for $\left|W_{\tau}\right| \leqslant 5$. Note that for large $W_{\tau}(> \pm 5)$ the $F\left(W_{\tau}\right)$ has a non-quadratic nature. A similar feature is also seen for 700-kHz data in Fig 3(b). At $1000 \mathrm{kHz}$ [(Fig. 3(c)] we see that the $F\left(W_{\tau}\right)$ is non-quadratic for all $W_{\tau}$, however, $R$ versus $W_{\tau}$ still obeys NEFR quite well [see Eq. (4) and Fig. 2(i)]. Thus, it seems that the quadratic symmetry of LDF isn't a prerequisite for validation of NEFR.

From the slope of the linear $R$ versus $W_{\tau}$ curve, using Eq. (5) we determine $\frac{1}{T_{\text {eff }}}$. The $T_{\text {eff }}$ associated with the nonequilibrium tunneling trajectories between the STM tip and the vibrating surface turns out to be very high, $T_{\text {eff }} \sim 10^{11} \mathrm{~K}$. It may be noted that $T_{\text {eff }}$ values determined for other NEQ systems have also been found to be quite high, $\sim 10^{6}-10^{16} \mathrm{~K}$
$[6,7,15,16]$. Instead of using the slope of $R$ versus $W_{\tau}$ curve as a measure of an effective temperature, it is convenient to describe it as an effective energy scale. We interpret the slope as a measure of the inverse of average dissipation $\left(\delta^{-1}\right)$ associated with our driven quantum tunneling system. In Fig. 4 we plot the slope $\left(\propto \delta^{-1}\right)$ versus $f$. Beyond $f=400 \mathrm{kHz}$, the dissipation associated with the quantum tunneling trajectory increases with the increasing barrier modulation frequency. The dissipation we have obtained using NEFR is a sensitive function of the modulation frequency. In Appendix C, we show $I-V$ curves measured of the Au film vibrating at different frequencies. The similarity of the $I-V$ curves shows that there are no changes in the density of states of the film due to the vibration. Hence the results we have obtained cannot be ascribed to any changes in the density of states of the film below the STM due to its vibration.

Historically the phenomenon of tunneling across a periodically modulated barrier came into focus with experiments showing the ionization of a neutral atom due to tunneling when placed in an alternating electric field $[18,36]$. It was found that the tunnel ionization probability depends on both the frequency and the amplitude of the drive. Within the

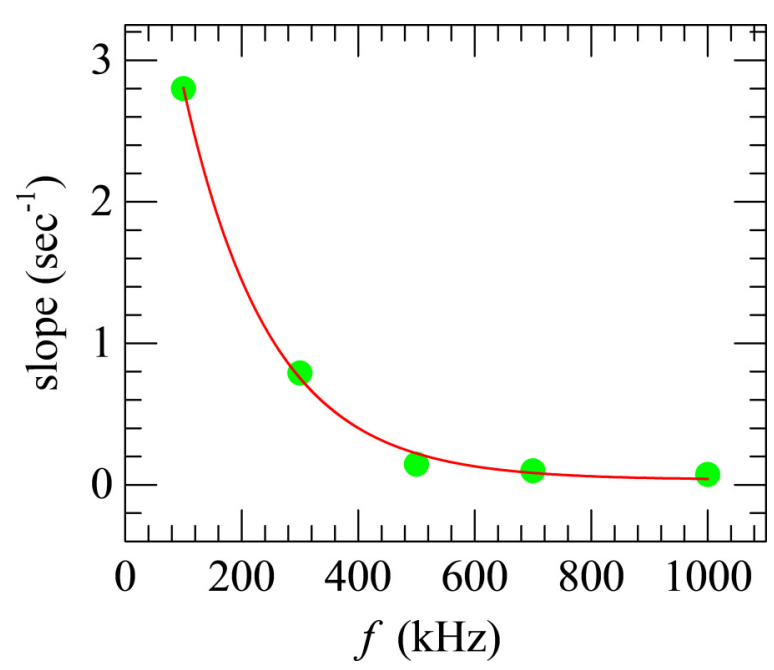

FIG. 4. Variation of slope $\left(\propto \frac{1}{T_{\text {eff }}}\right)$ with the frequency of the vibrating piezo. The solid line is a guide to the eye. 


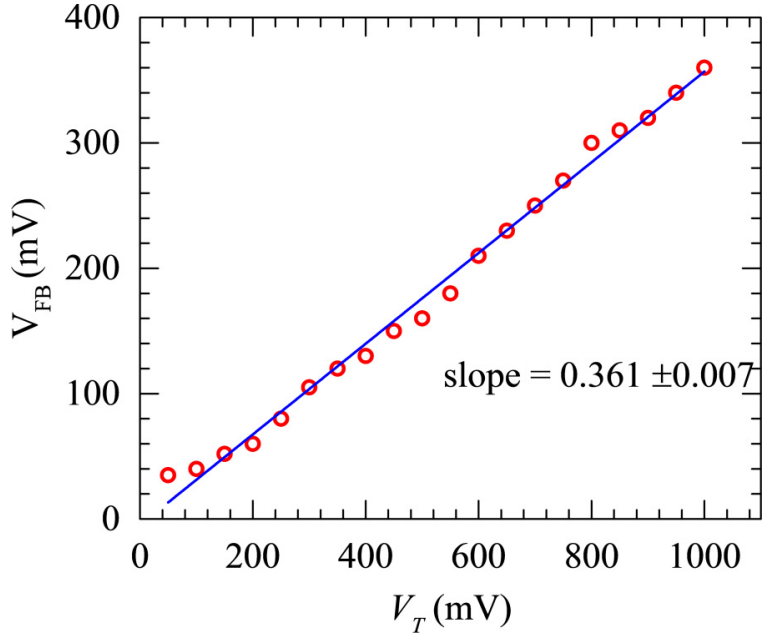

FIG. 5. Variation of the feedback signal with the tunneling voltage in the STM.

wave picture, it has been suggested that for the periodically modulated barrier, a part of the wave function tunnels across the barrier while a part of it remains back within the barrier. Thus, the part which is left back evolves within the modulated barrier while a part of it tunnels across the modulated barrier [22]. Typically for a barrier modulating at a frequency $f$, the solutions for the reflected and transmitted waves not only have the usual stationary wave solution, but also have waves which are reflected and transmitted at frequency $f$ [36-41]. To understand the positive and negative fluctuations in the tunneling event, we propose a scenario. Tunneling creates in its wake trapped electron waves within the barrier, with quantized energy levels [36]. Interaction of an impinging tunneling electron with the modulating barrier with states inside it can cause resonant excitations between levels within the barrier. Often this results in some loss of energy (dissipation), which is given up to the barrier as the electron tunnels through it, resulting in the positive entropy event. However, occasionally, due to the electron being temporarily trapped within the barrier, one would occasionally get reverse events. An incoming electron would temporarily see a higher barrier potential due to the added repulsive Coulombic potential due to the electrons temporarily inside the barrier. This temporary higher effective barrier height reduces the tunneling proba- bility. Furthermore, at the barrier, the incoming electron may also pick up energy from the states already present inside the barrier. Consequently, instead of tunneling through, the incoming electron may occasionally reflect back by picking up some energy from the barrier. This enables it to do work by moving opposite to the bias voltage direction, resulting in negative entropy events. For such a NEQ quantum mechanical tunneling electron system where energy is exchanged between the tunneling electron and the modulating barrier, the work fluctuations obey the NEFR.

In conclusion, unlike most earlier studies done for NEQ classical systems, here we show that the NEFR is valid even in the quantum regime, viz., for a dissipating quantum tunneling trajectory across a modulating barrier. The NEFR provides a useful way to measure and quantify dissipation associated with non-equilibrium dissipating trajectories in quantum mechanics. More future experimental and theoretical studies are needed to explore the complex behavior of NEQ quantum systems.

\section{ACKNOWLEDGMENTS}

S.S.B. acknowledges discussions with Prof. A. Sood of the Department of Physics, IISc Bangalore. S.S.B. would like to acknowledge funding support from IITK (IN) and DST-TSDP (IN) DST-SERB Imprint II (IN), Government of India.

\section{APPENDIX A: RELATION BETWEEN $V_{\mathrm{FB}}$ AND $V_{T}$}

Figure 5 shows that the feedback voltage has a linear relationship with the tunneling voltage, which shows that the $V_{\mathrm{FB}}$ is proportional to the tunneling signal $\left(V_{\mathrm{FB}} \propto V_{T}\right)$. From this we can see that the $V_{\mathrm{FB}}$ is also a faithful representation of the $V_{T}$.

\section{APPENDIX B: NON-SCALING FEATURE OF $R$ VERSUS $\boldsymbol{W}_{\tau}$ DETERMINED FOR DIFFERENT CHOICES OF $\tau$}

Recall Eq. (4) in the text which we mention below:

$$
R=\frac{1}{\tau} \ln \left(\frac{P\left(+W_{\tau}\right)}{P\left(-W_{\tau}\right)}\right)=s_{\tau}=W_{\tau}\langle s(t)\rangle .
$$

In Fig. 6(a) for 500-kHz time series data, we plot $\ln \left(\frac{P\left(+W_{\tau}\right)}{P\left(-W_{\tau}\right)}\right)$ versus $W_{\tau}$ for two very different values of choice of $\tau$. The
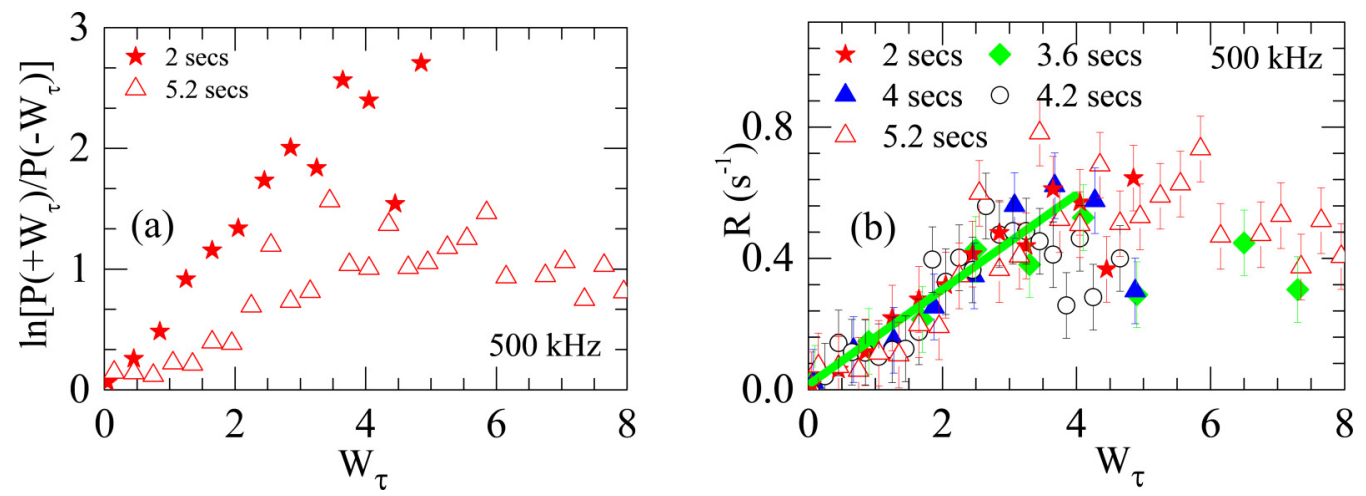

FIG. 6. (a) Unscaled data for $\tau=2$ and $5 \mathrm{sec}$. (b) Scaled data for $\tau=2,3.6,4,4.2$, and 5.2 sec for piezo vibrating frequency of $500 \mathrm{kHz}$. 
figure clearly shows that the data for 2 and 5.2 sec do not overlap. Figure 6(b) shows that the analysis becomes independent of the choice of $\tau$ and all the data scale onto a single curve by plotting $R$ versus $W_{\tau}$ [which follows from Eq. (B1)]. We see that within $0<W_{\tau}<4$, the best-fit curve through the scaled data in the plot in Fig. 6(b) is a straight line with a unique slope.

\section{APPENDIX C: $I-V$ CURVES}

We show in Fig. 7 that the local $I-V$ of the Au film (on top of the piezo) measured with the STM for different vibrating frequencies are identical, suggesting that the states of the $\mathrm{Au}$ film into which the electrons are tunneling into are identical at different frequencies. Hence, any change in the density of electronic states of the film is not responsible for the observed features in the $V_{\mathrm{FB}}(t)$.

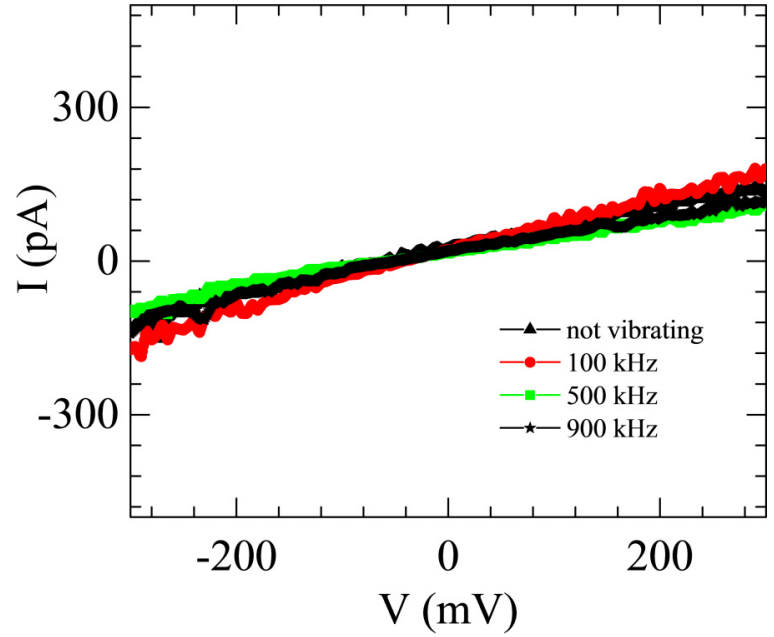

FIG. 7. Comparison of the $I-V$ curves on a piezo vibrating at different frequencies.
[1] Carlos Bustamante, Jan Liphardt, and Felix Ritort, Phys. Today 58(7), 43 (2005).

[2] J. M. R. Parrondo, C. Van den Broeck, and R. Kawai, New J. Phys. 11, 073008 (2009).

[3] D. J. Evans and D. J. Searles, Phys. Rev. E 50, 1645 (1994).

[4] G. Gallavotti and E. D. G. Cohen, Phys. Rev. Lett. 74, 2694 (1995); J. Stat. Phys. 80, 931 (1995).

[5] Shragel and Chou, J. Stat. Phys. 137, 165 (2009).

[6] S. Majumdar and A. K. Sood, Phys. Rev. Lett. 101, 078301 (2008).

[7] K. Feitosa and N. Menon, Phys. Rev. Lett. 92, 164301 (2004).

[8] D. M. Carberry, J. C. Reid, G. M. Wang, E. M. Sevick, Debra J. Searles, and Denis J. Evans, Phys. Rev. Lett. 92, 140601 (2004).

[9] G. M. Wang, E. M. Sevick, Emil Mittag, Debra J. Searles, and Denis J. Evans, Phys. Rev. Lett. 89, 050601 (2002).

[10] N. Garnier and S. Ciliberto, Phys. Rev. E 71, 060101 (2005).

[11] X. D. Shang, P. Tong, and K.-Q. Xia, Phys. Rev. E 72, 015301 (2005).

[12] S. Ciliberto and C. Laroche, J. Phys. IV France 8, Pr6-215 (1998).

[13] S. Ciliberto, N. Garnier, S. Hernandez, C. Lacpatia, J.-F. Pinton, and G. R. Chavarria, Phys. A (Amsterdam, Neth.) 340, 240 (2004).

[14] M. M. Bandi and C. Connaughton, Phys. Rev. E 77, 036318 (2008).

[15] W. I. Goldburg, Y. Y. Goldschmidt, and H. Kellay, Phys. Rev. Lett. 87, 245502 (2001).

[16] B. Bag, G. Shaw, S. Banerjee, S. Majumdar, A. Sood, and A. Grover, Sci. Rep. 7, 5531 (2017).

[17] Jan Gieseler, Romain Quidant, Christoph Dellago, and Lukas Novotny, Nat. Nanotechnol. 9, 358 (2014).

[18] Quantum Mechanics: Non-Relativistic Theory, edited by L. D. Landau and E. M. Lifshitz (Pergamon, New York, 1977).
[19] S. Kohler, T. Dittrich, and P. Hanggi, Phys. Rev. E 55, 300 (1997).

[20] T. Dittrich, B. Oelschlägel, and P. Hänggi, Europhys. Lett. 22 5 (1993).

[21] B. Oelschlägel, T. Dittrich, and P. Hänggi, Acta Phys. Pol. B 24, 845 (1993).

[22] C. K. Andersen and K. Mølmer, Phys. Rev. A 87, 052119 (2013).

[23] A. O. Caldeira and A. J. Leggett, Phys. Rev. Lett. 46, 211 (1981).

[24] A. J. Leggett, S. Chakravarty, A. T. Dorsey, M. P. A. Fisher, A. Garg, and W. Zwerger, Rev. Mod. Phys. 59, 1 (1987); 67, 725(E) (1995).

[25] P. Hänggi, P. Talkner, and M. Borkovec, Rev. Mod. Phys. 62 251 (1990).

[26] U. Weiss, Quantum Dissipative Systems (World Scientific, Singapore, 1993).

[27] K. Baumann, C. Guerlin, F. Brennecke, and T. Esslinger, Nature (London) 464, 1301 (2010).

[28] J. Klinder, H. Keßler, M. Wolke, L. Mathey, and A. Hemmerich, Proc. Natl. Acad. Sci. USA 112, 3290 (2015).

[29] E. Altman, L. M. Sieberer, L. Chen, S. Diehl, and J. Toner, Phys. Rev. X 5, 011017 (2015).

[30] M. Fitzpatrick, N. M. Sundaresan, A. C. Y. Li, J. Koch, and A. A. Houck, Phys. Rev. X 7, 011016 (2017).

[31] S. R. K. Rodriguez, W. Casteels, F. Storme, N. C. Zambon, I. Sagnes, L. L. Gratiet, E. Galopin, A. Lemaitre, A. Amo, C. Ciuti, and J. Bloch, Phys. Rev. Lett. 118, 247402 (2017).

[32] J. M. Fink, A. Dombi, A. Vukics, A. Wallraff, and P. Domokos, Phys. Rev. X 7, 011012 (2017).

[33] Dibya J. Sivananda, Amit Banerjee, and S. S. Banerjee, J. Appl. Phys. 122, 114302 (2017).

[34] H. Touchette, Phys. Rep. 478, 1 (2009).

[35] Nitin Kumar, Sriram Ramaswamy, and A. K. Sood, Phys. Rev. Lett. 106, 118001 (2011). 
[36] L. V. Keldysh, Sov. Phys. JETP 20, 1307 (1965) [JETP 47, 1945 (1964)].

[37] Z. S. Gribnikov and G. I. Haddad, J. Appl. Phys. 96, 3831 (2004).

[38] M. Grifoni and P. Hänggi, Phys. Rep. 304, 229 (1998).
[39] F. Grossmann, T. Dittrich, P. Jung, and P. Hänggi, Phys. Rev. Lett. 67, 516 (1991).

[40] F. Grossmann, P. Jung, T. Dittrich, and P. Hänggi, Z. Phys. B 84, 315 (1991).

[41] L. Wang and J. Shao, Phys. Rev. A 49, R637 (1994). 